\title{
Pathology \\ HISTOPATHOLOGICAL SPECTRUM OF APPENDICITIS IN A TERTIARY HEALTH CARE CENTER
}

\section{Dr. A. R Jaswanthini \\ Mr. Amitesh Krishna S* $^{*}$ \\ ABSTRACT}

Assistant professor, Department of Pathology, Government Medical College, Omandurar Government Estate, Chennai 600002 II year MBBS Student, Government Medical College, Omandurar Government Estate, Chennai $600002 *$ Corresponding Author

INTRODUCTION: Appendix in a finger like projection situated just near the caecum. It is located in the right iliac fossa of the abdomen. The most common pathology which arises is appendicitis and is mostly treated surgically. Although appendix is considered to be a vestigial organ, it accounts for immune function upto some extent

OBJECTIVES: To study and analyse the various histopathological forms of appendicitis

MATERIALS AND METHODOLOGY: A descriptive study was undertaken to analyse the different histopathological forms of appendicitis over a period of one year in the Department of Pathology, Government Medical College, Omandurar Government Estate. The acquired data was fed into Microsoft Excel and analysis was done.

RESULT: A total of 100 specimens were analysed during the study period. Maximum number of cases ( $62 \%$ of cases) were reported amongst females. The most common age group which was affected was 9 years to 19 years. The most common histopathological form of appendicitis was analysed to be acute appendicitis ( $76 \%$ of cases)

The statistical data for various histo pathological types of appendicitis are as follows

Subacute appendicitis -10 cases ; Acute appendicitis -76 cases ; Chronic appendicitis -13 cases ; Granulomatous appendicitis -1 case.

CONCLUSION: The incidence of appendicitis is higher in the second decade of life and is remarkably high amongst females. Among the pathological types, incidence of acute appendicitis is the highest and that is seen in the second decade of life.

KEYWORDS : Acute appendicitis, granulomatous appendicitis, chronic appendicitis

\section{INTRODUCTION}

The appendix was first described by Berengario Di Carpi in 1521 Vermiform means worm like, appendix is referred to as a 3inch earthworm due to its size and hence the name. Appendix is considered to be a vestigial organ of the human body. Anatomically appendix in located in the right lower quadrant of the abdomen. It is described as a finger like projection near the ileo-caecal junction. The appendix of a child is bigger than the appendix of an adult. Although it is a vestigial organ, it accounts for immunity especially in people who have been exposed to radiation ${ }^{2,8}$. In a healthy human being the appendix plays a major role in controlling the population of the gut flora. ${ }^{2,9}$ It has a life time risk of $6 \%{ }^{3,10}$ it has an incidence of 86 per 100,000 population per year ${ }^{3,1}$

The histology of the appendix of a child resembles a lymphoid tissue and hence helps in immunity ${ }^{2}$. Acute appendicitis is one of the most common surgical causes of acute abdomen. The lifetime risk for appendicitis is $7 \%$; commonly occurring in adolescents and young adults.

Appendix does not draw the interest of a physician unless it manifests as one or the other pathological form. The most common pathology of appendix is appendicitis and the latter is the most common cause for acute abdominal colic. The appendicitis is generally managed surgically; medical management of appendicitis comes into play only if surgery is contraindicated. Despite the advancements in technology and improvement in diagnostic tests, histopathological analysis still holds the gold standard position for classifying the pathological and clinical type of appendicitis. This study aims at the histopathological variants of appendicitis which would provide a deeper insight on its pathogenesis and hence its management. Some studies have shown that appendicitis is more common in 10-29 years of age group. ${ }^{3,5}$ Males are more susceptible than females.

\section{AIMS AND OBJECTIVES}

This study aims at analysing the histopathological variants of appendicitis in a tertiary care hospital

\section{MATERIALSAND METHODOLOGY}

This study is a descriptive study. All the appendicectomy specimens which came to the Department of Pathology, Omandurar Government Estate were taken for analysis.

A total of 100 appendicectomy samples were collected and were studied for a period of 1 year. The specimens were collected from the Department of General Surgery after appendicectomy.
The specimens were subjected to $10 \%$ formalin fixation for 24 hours The fixed specimens were then sent for paraffin embedding.

Small sections were cut out from the paraffin block and specimen were routinely stained using hematoxyl in and eosin.

The stained specimens were studied under a compound microscope with low and high power lenses.

Minimum of two representative samples were taken from each specimen.

The collected data were fed into Microsoft Excel Spreadsheet software and analysis was done by calculating percentage and representative tables and figures.

\section{RESULTS}

APPENDICITIS - SEX VARIATION

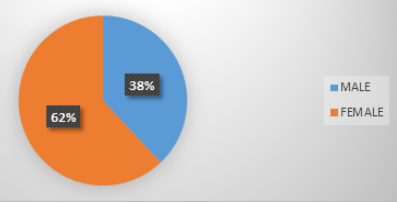

Pie chart 1. Incidence of appendicitis in different sexes

Table 1: Sex distribution of appendectomy

\begin{tabular}{|c|c|}
\hline MALE & FEMALE \\
\hline $38 \%$ (38 cases) & $62 \%(62$ cases $)$ \\
\hline
\end{tabular}

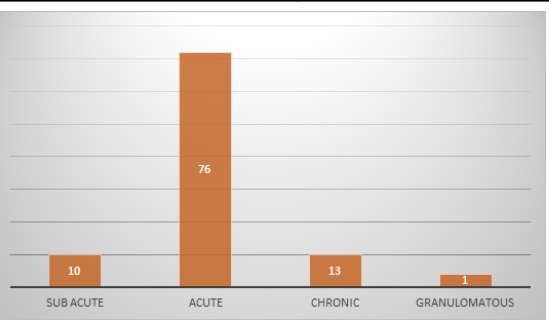

Graph 1 Incidence of different pathological types of appendicitis 
Table 2: Histo logical spectrum of appendicitis

\begin{tabular}{|l|l|l|l|}
\hline SUBACUTE & ACUTE & CHRONIC & GRANULOMATOUS \\
\hline $\begin{array}{l}10 \% \\
(10 \text { cases })\end{array}$ & $\begin{array}{l}76 \% \\
(76 \text { cases })\end{array}$ & $\begin{array}{l}13 \% \\
(10 \text { cases })\end{array}$ & $1 \%(1$ cases $)$ \\
\hline
\end{tabular}

Out of the 100 cases which appeared in the last one year 72 cases $(72 \%)$ belonged to the acute type of appendicitis, the gangrenous type of appendicitis was seen only in 1 patient $(1 \%)$ while subacute and chronic type of appendicitis had an equal distribution of 10 patients each $(10 \%)$ [graph 1, table 2]

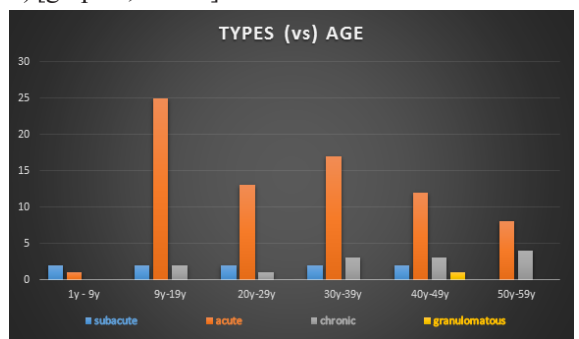

Graph 2. Incidence of different types of appendicitis in different age groups

Table 3: Different histological types of appendicitis in different age group

\begin{tabular}{|l|l|l|l|l|}
\hline Type/age & Subacute & Acute & Chronic & Granulomatous \\
\hline 1 to 9 & 2 & 1 & 0 & 0 \\
\hline 9 to 19 & 2 & 25 & 2 & 0 \\
\hline 20 to 29 & 2 & 13 & 1 & 0 \\
\hline 30 to 39 & 2 & 17 & 3 & 0 \\
\hline 40 to 49 & 2 & 12 & 3 & 1 \\
\hline 50 to 59 & 0 & 8 & 4 & 0 \\
\hline
\end{tabular}

Most of the patients who have registered for appendicitis fall under the category of 10 years to 19 years $(29 \%)$ and the maximum number of cases is categorised as acute appendicitis 25 cases $(86.2 \%)$. The number of subacute cases was distributed equally among all the age groups, however there were no cases of subacute appendicitis in the age group between 50 years and 59 years. The peak incidence of chronic appendicitis was observed between 50 years and 59 years (30.76\%). When compared to all the other histopathological types, there was only 1 case of granulomatous appendicitis in the age group of 40 years to 49 years. (graph 2, Table3)

\section{DISCUSSION}

Appendicitis is the most common pathology This study illustrates the incidence of different pathological types of appendicitis in different age groups.

SUB ACUTE APPENDICITS: the histopathology is very similar to that of acute appendicitis except for the neutrophil infiltrate is restricted to the mucosal and submucosal layers of the wall.

ACUTE APPENDICITIS: In acute appendicitis subserosal vessels are congested and there is a modest perivascular neutrophilic infiltrate within all the layers of the wall. The surface looks dull and erythamatos. Hallmark of acute appendicitis is neutrophilic infiltration of the muscularis propria. (image 1 and 2) In some cases, aggregates of neutrophils are also seen in lumen(image 3 )

CHRONIC APPENDICITS: it is characterised by fibrosis of the wall of the organ and lymphatic infiltration. In severe cases, excudate containing lymphocytes might be found in the lumen. In some cases, necrosis might be found due to hyper congestion. (image 4)

GRANULOMATOUS APPENDICITS: This is caused by an underlying systemic granulomatous inflammation like sarcoidosis, amyloidosis etc. A typical granulomatous necrotic image is present. It is a focal inflammation consisting of a microscopic aggregation of macrophages that are transformed into epithelium like cells surrounded by a collar of mononuclear leukocytes and plasma cells. The inflammation is persistent through all the layers of the wall. (image 5)

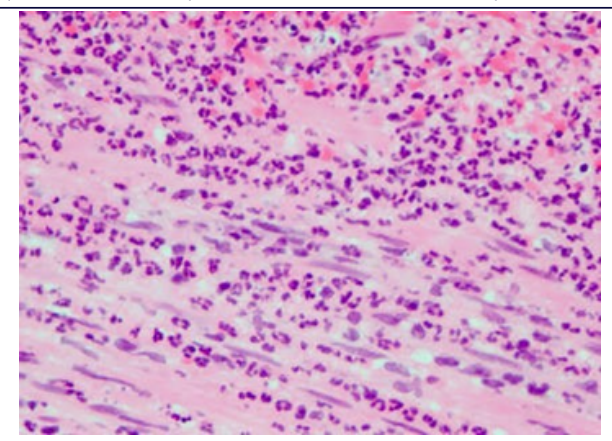

Image 1: Histo pathology of Acute Appendicitis

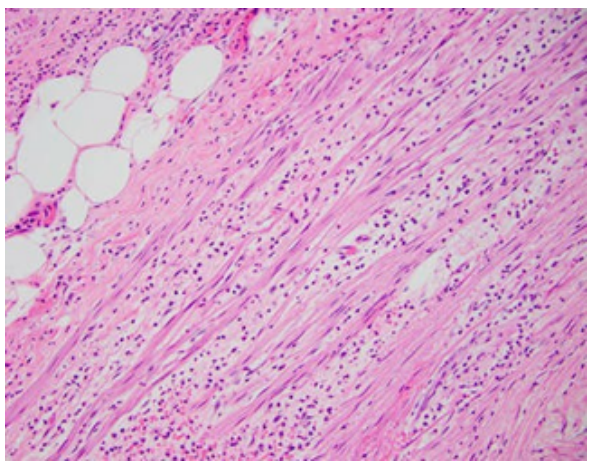

Image 2: Histopathology of acute appendicitis showing neutrophilic infiltration in the wall of the organ

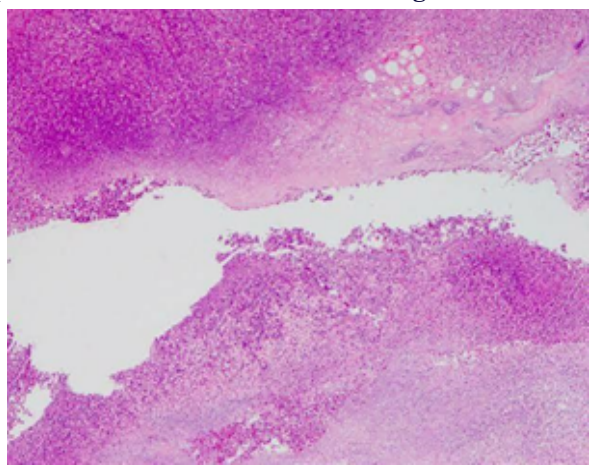

Image 3: Histopathology of acute appendicitis showing intraluminal neutrophilic infiltration

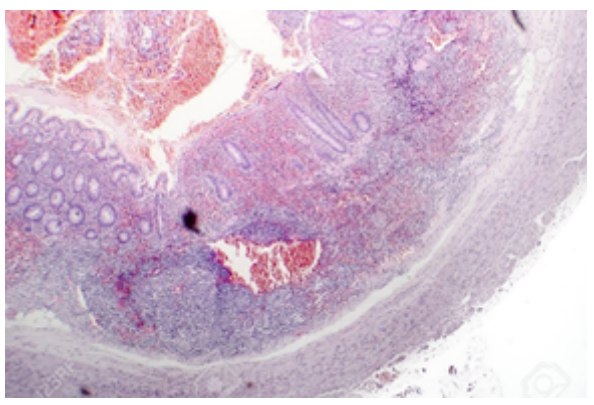

Image 4: Histopathological view of chronic appendicitis

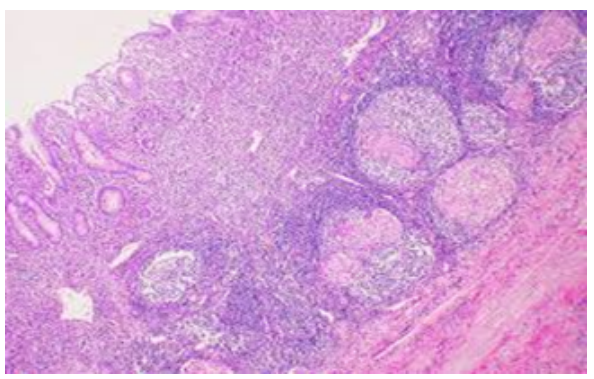

Image 5: Histopathological view of granulomatous appendicitis 


\section{CONCLUSION:}

The incidence of appendicitis is higher in the second decade of life and is remarkably high amongst females. Among the pathological types, incidence of acute appendicitis is the highest and that is seen in the second decade of life. The mean age of this study is 27.6 years. Hall marks of acute appendicitis that is neutrophil infiltration were seen in almost all the cases. In all the chronic appendicitis, fibrosis of the wall was clearly seen.

\section{REFERENCES:}

1. Mishra RK, Goel P, Sharma R, Sharma AC. The Epidemiology of Appendicitis and Mishra RK, Goel P, Sharma R, Sharma AC. The Epidemiology of Appendicitis and
Appendectomy in India : An Observational Study. 2017;3(5):381-387. doi:10.21276/ijmrp.2017.3.5.073

2. George JR, George JR. Histological observations and its variations in appendix at different stages of life. 2016;4(6):2324-2327.

3. Chaudhari YP, Jawale PG. $\mathrm{t}$ of a tertiary care hospital : A descriptive study. 2015;2(November):768-770.

4. Shrestha R, Sr R, Tiwari M. Histopathologic analysis of appendectomy specimens. 2012;2:215-219.

5. Macklin CP, Radcliffe GS, Merei JM, Stringer MD. A prospective evaluation of modified Alvarado scores for acute appendicitis in Children. Ann R Coli Surg modified Alvarado scors
Eng11997; 79:203-205.

6. Marudanayagam R, Williams G, Rees B. Review of the pathological results of 2660

7. Turner JR. The Gastrointestinal tract, In: Kumar,Abbas,Fausto (eds). Robins and Cotran Pathologic basis of disease. 8th edn. Saunders: Philadelphia; 2010. pp870-1.

8. Buschard and Kjaeldguard. Investigations and Analysis of the position, fixation, length \& embryology of the vermiform appendix. Acta Chir Scand. 1992;139:293-8.

9. Elias EG, Hults R. Congenital absence of Vermiform Appendix. Arch Surg. $1967 ; 95(2): 257-8$

10. Hartwig K, Karl S, Jon AS, Egil A, Arne N, Tone HL et al. Incidence of Acute Non perforated and perforated appendicitis: Age specific and Sex specific analysis. World J
Surg. 1997; 21:313-317.

11. Addiss DG, Shaffer N, Fowler BS, Tauxe RV. The epidemiology of Appendicitis and appendicectomy in the United States. Am J. Epidemiol. 1990; 132(5):910-25. 